\title{
What happens after referral for sedation?
}

IN BRIEF
- A second stage treatment-planning
visit increases the likelihood of that
individual attending a sedation treatment
appointment and has cost savings.
- Older dentally anxious patients are more
likely to complete treatment.
- People with mental health disorders are
less likely to attend and may require
additional support.
A significant number of appointments are
wasted due to patient non-attendance.

wasted due to patient non-attendance.

\author{
C. A. Boyle, ${ }^{1}$ T. Newton, ${ }^{2}$ L. J. Heaton, ${ }^{3}$ S. Afzali ${ }^{4}$ and P. Milgrom ${ }^{5}$ \\ VERIFIABLE CPD PAPER
}

\begin{abstract}
Objective To follow up 100 referrals to the sedation clinic, examining dental anxiety and background of patients, and to assess how many patients attended for treatment planning, initial treatment and how many completed treatment, and describe the characteristics of each. For those who attended for initial treatment, to investigate which type of sedation they received and the level of clinician they saw. Design Descriptive, cross-sectional survey and review of case notes. Subjects and methods Subjects were 100 consecutive new patients to the Department of Sedation and Special Care Dentistry at Guy's and St Thomas NHS Foundation Trust. The notes were analysed by an experienced member of staff (CAB) and data entered into an Excel spreadsheet and an SPSS data file created. These data were merged with a dataset containing their responses to the initial questionnaire and medical history for analysis. Results of the 100 patients initially referred, 72 attended the treatment planning session, 66 of the $72(92 \%)$ attended for initial dental treatment, and 33 of 66 (50\%) completed treatment. Dental Fear Survey (DFS) scores were related to attendance at the initial treatment visit but not to completion of treatment. Only 33 of 100 referred patients completed treatment. Conclusions Attendance for treatment planning and initial treatment was high. Attendance is related to fear and mental health. Overall completion of treatment from referral was $33 \%$.
\end{abstract}

\section{INTRODUCTION}

Avoidance of dental care due to fear is a common problem: approximately $25 \%$ of adults in the UK do not seek treatment for painful dental conditions due to dental fear. ${ }^{1}$ This avoidance of care typically leads to deterioration in oral health such that more invasive treatment is necessary when

\footnotetext{
${ }^{1 *}$ Consultant in Special Care Dentistry, Department of Sedation and Special Care Dentistry, King's College London Dental Institute at Guy's, King's College and St Thomas' Hospitals, Floor 26, Tower Wing, London, SE1 9RT, UK; ${ }^{2}$ Professor of Psychology as Applied to Dentistry, King's College London, Oral Health Services Research \&t Dental Public Health, King's College Hospital, Caldecot Road, London, SE5 9RW, UK; ${ }^{3}$ Acting Assistant Professor, Dental Fears Research Clinic, Department of Dental Public Health Sciences, Box 357475, University of Washington, Seattle, WA 98195-7475, USA; ${ }^{4}$ Student of Dental Hygiene and Therapy, Faculty of Health Care, Utrecht University of Applied Sciences, Bologna-

laan 101, 3584 CJ Utrecht, The Netherlands; ${ }^{5}$ SAAD Visiting Professor of Anxiety and Pain Management, Department of Sedation and Special Care Dentistry,

King's College London Dental Institute at Guy's, King's

College and St Thomas' Hospitals, Floor 26, Tower Wing, London, SE1 9RT, UK

${ }^{*}$ Correspondence to: $\operatorname{Dr}$ Carole A. Boyle

Email: carole.boyle@kcl.ac.uk;

Tel: +44 (0)2071886074
}

\section{Online article number E22}

Refereed Paper - accepted 19 February 2010

DOI: $10.1038 /$ sj.bdj.2010.502

${ }^{\circ}$ British Dental Journal 2010; 208: E22 dental care is finally sought. ${ }^{2}$ Fearful individuals often find themselves in a "cycle of avoidance' involving extensive periods lacking regular care interspersed with painful dental symptoms and invasive, potentially traumatic treatment, which further reinforces avoidance of care. ${ }^{3}$

Individuals who delay seeking treatment due to fear are often referred to receive treatment under sedation or general anaesthesia through specialist dental care. According to 2003 National Health Service (NHS) estimates, over £6 million was spent on treatment under sedation alone in primary care. This estimate does not take into account the costs of those treated in secondary care or in the salaried services, or the time lost from work and other activities due to dental problems.

Little is known about the impact of sedation on long-term dental treatment-seeking behaviour in a population of individuals referred for dental treatment under sedation. Berggren followed 84 patients for two years after 'full oral rehabilitation' using either general anaesthesia (GA) or behaviour therapy (BT) to manage dental fear. ${ }^{4}$ While $70 \%$ of GA patients (28 of 40) were receiving regular dental care two years after receiving dental treatment, 93\% (41 of 44) of BT patients were receiving regular care, representing a significant increase in regular treatment-seeking for those treated behaviourally over treatment with sedation. A more recent study also found significantly more regular dental attendance in individuals receiving BT compared to GA ten years after treatment. ${ }^{5}$

In these studies, patients attended a specialised dental fears treatment centre that, while offering treatment under sedation, also places an emphasis on behavioural strategies for managing dental fear. Patients who completed these studies were randomly assigned to either behavioural or pharmacological treatment modalities, and thus were willing to accept either type of treatment. What is not known is how individuals referred specifically for dental treatment under sedation seek and receive treatment after sedation, and how patient characteristics impact on their later use of dental services.

\section{OBJECTIVE}

The current study provides follow-up data on individuals referred specifically 
for dental treatment under sedation, and also presents information about medications used and who the patients saw for treatment.

\section{DESIGN}

This is a descriptive, cross sectional study with longitudinal follow-up.

\section{SETTING}

The study was conducted in the Department of Sedation and Special Care Dentistry at Guy's and St Thomas NHS Foundation Trust. The study was reviewed and approved by the Research Ethics Committee of St Thomas' Hospital. The survey was confidential and the informed consent of each participant was obtained.

\section{SUBJECTS AND METHODS}

The study subjects were 100 consecutive new patients being evaluated for sedation at a sedation assessment clinic in the Department of Sedation and Special Care Dentistry who were referred because their general dental practitioner has been unable to provide dental care due to their anxiety. The initial study was carried out between January and June 2007. The notes for these patients were recalled and examined in June 2009.

A 34-item written questionnaire was administered after confirmation that the patients were able to read and write English and were willing to answer questions. The questionnaire included demographic information, self-reported oral health (four-point Likert-like scale ranging from 'poor' to 'excellent'), self-reported dental attendance (five-point Likert-like scale ranging from 'only when I need to' to 'more often than every six months') and reasons for visits to the dentist (emergency treatment or routine checkup, cleaning or filling), anxiety regarding dental injections (five items ranging from 'not at all true' to 'absolutely true'), ${ }^{6}$ and a general measure of dental fear (Dental Fear Survey (DFS), 20 items, five-point scales) as well as the sub-scores on the DFS for anticipation, specific fears and physiology. ${ }^{7}$ Additional items were included in the questionnaire to capture other aspects of dental anxiety. The questionnaire was pretested on patients attending other clinics before use.

\section{Table 1 Attendance at sedation clinic at Guy's Hospital, London}

\begin{tabular}{|l|l|}
\hline Stage of treatment & Attendance/type of treatment/provider \\
\hline Attended clinic for treatment planning & $\begin{array}{l}\text { Yes: } 72(72 \%) \\
\text { No: } 19\end{array}$ \\
\hline Attended initial treatment visit & Yes: $66(91.7 \%)$ \\
No: 6
\end{tabular}

Information taken from the patients' medical records included American Society of Anesthesiologists Classification (ASA), previous sedation or general anaesthesia for dentistry, alcohol and tobacco use, and a note made of whether the patient had a mental health condition, the nature of the condition, and any medication taken.

Data taken from notes included whether or not the patient attended for treatment planning. If the patient did attend, the following information was recorded:

- Which type of sedation and the amount of drug (or none) used at the first and each subsequent session

- Who the patient saw for treatment staff, postgraduate or undergraduate student for each appointment

- Whether the dental care treatment plan was completed.

The patient records were obtained in batches and no more than ten records were reviewed at one session to avoid errors from fatigue.

In order to assess the reliability of the record review, 14 records were randomly selected from among the original population using the RAND function of Excel and re-reviewed after four months by the same clinician without consultation to the original data extraction. Kappa was calculated as a measure of inter-rater reliability. The Kappa values ranged from 0.69 to 1 , indicating the reliability was good. The lowest value was for the completed treatment variable.

The data were entered into Excel, edited, and read into SPSS (Statistical Package for the Social Sciences, version 16 for Mac). This dataset was then merged with the original dataset containing questionnaire responses and items from the medical history. The merged dataset was analysed using SPSS.

\section{HYPOTHESES}

As fear level and previous attendance were the best predictors of referral for sedation in this setting, ${ }^{8}$ it is hypothesised that these factors will be related to attending and following through with treatment. While dental fear is typically associated with avoidance of regular care, it is believed that individuals with higher fear will attend sedation appointments as many fearful patients see sedation as the solution to their fear avoidance. On the other hand, co-morbid mental health problems will be associated with failing to follow through because individuals with mental health problems have fewer coping skills and are less able to negotiate routine activities.

\section{MAIN OUTCOME MEASURES}

The main outcome measures were 1) attending the treatment planning appointment; 2) attending the initial treatment session; and 3) completing all treatment prescribed.

\section{RESULTS}

One hundred consecutive new patients (77\% female, mean age 36.5 years, range 16 to 67$)$ participated in the study. The overall DFS mean score was 69.8, SD 18.9, range 20-97. DFS sub-score means were anticipation (mean 9.9, SD 3.0), specific 
fears (mean 42.0, SD 12.7), and physiology (mean 17.7, SD 5.3). Thirteen of the 100 patients had a history of mental health problems.

\section{Treatment planning appointment}

Nearly three-quarters $(72 / 100)$ of the referred patients attended the treatment planning appointment. Contrary to our hypotheses, there were no differences in DFS scores (attenders mean DFS 69.6, SD 18.5 versus non-attenders mean DFS 70.5, SD 20.6) or DFS sub-scores for anticipation, specific fears, or physiological upset between the groups. Similarly there was no difference in attendance between those with and without other mental health conditions. Among attenders, 10/72 (13.9\%) had a comorbid mental health condition versus 3/28 (10.7\%) of non-attenders. There were no differences between the two groups for age, education level, self-reported oral health or previous attendance pattern.

\section{Initial treatment session}

Table 1 shows the types of treatments provided at the initial treatment session and who provided the treatment. Nearly all patients seen for treatment planning attended the initial treatment visit (66/72, 91.7\%). Consistent with the hypothesis, those patients who attended had higher DFS scores than those who did not attend (attenders mean DFS 70.8, SD 17.8 versus non-attenders mean DFS 59.0, SD 23.0) but only the DFS sub-score for anticipation was significantly different (attenders mean DFS 10.3, SD 2.8 versus non-attenders mean DFS 6.7, SD 3.3, $\mathrm{t}=2.9, \mathrm{p}=0.005)$. There were no significant differences between attenders and non-attenders in sub-scores for specific fears or physiological arousal, although the differences were in the expected direction. Attendance rates were 95.2\% for those without mental health problems and 70\% for those with problems (Exact Test, $p=0.032$ ). There were no differences among the two groups for age, education level, self-reported oral health or previous attendance pattern.

The predominant treatment at the initial session was intravenous sedation (Table 1) while fewer patients received other treatment modalities. The overall pattern of DFS scores was highest for intravenous sedation patients (mean DFS 73.3, SD 16.6) but there were no significant differences between the groups. None of the background variables studied was related to the choice of treatment. Thus it is likely the clinicians used another criterion besides fear, perhaps the extent of treatment needed or the clinician's assessment of the invasiveness of the treatment, in assigning patients to treatments.

\section{Treatment completion}

Among those who initiated treatment, 33 of 66 (50\%) completed all treatment. The number of visits ranged from 1 to 17 with the typical patient having 4 treatment visits. DFS mean scores were 71 for both groups. Neither were there differences in mental health status between the groups. Among the background variables, the only significant finding was that completers tended to be 38 years old and non-completers were younger (chi square 3.98, df $1, p=0.046)$. Only 33 of the original 100 patients referred for sedation completed all treatment.

\section{DISCUSSION}

By NHS estimates, more than £6 million per year is spent on sedation for dentistry in primary care. In order to prioritise limited treatment resources, it is critical to understand the characteristics of individuals who follow through with treatment recommendations for dental treatment under sedation.

Previous research ${ }^{8}$ showed that those individuals who were more fearful were the ones who were referred for dental treatment under sedation. None of the variables measured, however, could differentiate between those who actually followed through with the referral by attending the treatment planning session and those who did not. At this stage, factors such as staff-patient interactions, convenience, time lost from work or expense in returning for treatment are not known.

What is known from this study is that, among those who attended the treatment planning session, adherence with the initial treatment was very high. More fearful individuals were more likely to attend the treatment planning sessions, suggesting a good match between patient expectations and needs and the treatment offered. Allen and Girdler found that over two-thirds of patients surveyed in an emergency dental clinic reported at least some dental anxiety, and over half said they would prefer to have treatment done under sedation if it were available. ${ }^{9}$ Chanpong and colleagues found that 31\% of highly fearful Canadians surveyed 'were definitely interested' in having dental treatment under sedation..$^{10}$ Clearly, having dental treatment done under sedation is an attractive option for many fearful individuals.

The higher non-attendance rate among those with mental illness suggests a point where intervention may be helpful. Individuals with mental health problems face additional barriers to dental care than those without such problems. Cutler noted that many with mental illnesses, particularly mood disorders, are unable to follow through with adequate self-care behaviours. ${ }^{11}$ In the United States, individuals with mental health issues are recognised by the Special Care Dentistry Association (SCDA) as a population requiring additional treatment considerations in the same spirit as the treatment of those with physical disabilities. ${ }^{12}$ Further exploration of how to best help these individuals is required. Since mood and anxiety disorders predominate in this population and skills in coping may be lacking, strategies to help these individuals become more comfortable in this environment may be helpful. Similarly, working with caregivers or other family members may improve attendance.

The low rate of completion of needed treatment is disheartening. Other than age, none of the factors studied differentiated between the two groups. Again, unmeasured are clinician-patient interactions at this stage or factors such as convenience, time lost from work or expense in returning for treatment. Clinician type (Table 1) was unrelated to completion of treatment; however, there was a trend toward lower completion rates among patients treated by staff $(6 / 17,35.3 \%)$ versus undergraduate $(6 / 13,46.2 \%)$ or postgraduate $(21 / 36$, $58.3 \%)$ students. This may be a factor of case assignment and complexity but may also reflect continuity of care, with postgraduates in this department more likely to see patients for all their dental care. Neither fear levels nor mental health status was related to assignment of care to a particular type of provider. 


\section{CONCLUSIONS}

Individuals referred for dental treatment under sedation followed through with treatment planning and initial treatment sessions at high rates. Higher dental fear was associated with better attendance, suggesting that those with high fear see treatment under sedation as an attractive option. Those with mental health problems followed through at a lower rate, suggesting more barriers to treatment completion for this population. Only one third of those referred overall, however, completed the entire treatment plan. These results suggest that high dental fear may be a driving factor behind initiating, but not necessarily finishing, dental care. Further work is needed on how to motivate those with mental health problems to initiate treatment and those referred in general to complete this necessary dental treatment.

This research was supported, in part, by a grant from the Society for the Advancement of Anaesthesia in Dentistry (SAAD) and grant no. 1 K23DE019202 to Dr Heaton from the National Institute of Dental and Craniofacial Research, National Institutes of Health, Bethesda, MD, USA. We acknowledge the advice of Dr David Craig of the King's College Dental Institute and Emeritus Professor Isaac Marks of the Institute of Psychiatry in carrying out this study.

1. Nuttall N M, Bradnock G, White D, Morris J, Nunn J. Dental attendance in 1998 and implications for the future. Br Dent J 2001; 190: 177-182.

2. McGrath C, Bedi R. The association between dental anxiety and oral health-related quality of life in Britain. Community Dent Oral Epidemiol 2004; 32: $67-72$.

3. Armfield J M, Stewart J F, Spencer A J. The vicious cycle of dental fear: exploring the interplay between oral health, service utilization and dental fear. BMC Oral Health 2007; 7: 1.

4. Berggren U. Long-term effects of two different treatments for dental fear and avoidance. J Dent Res 1986; 65: 874-876.
5. Hakeberg M, Berggren U, Carlsson S G, Gröndahl H G. Long-term effects on dental care behaviour and dental health after treatments for dental fear. Anesth Prog 1993; 40: 72-77.

6. Milgrom P, Coldwell S E, Getz T, Ramsay D S, Weinstein P. Four dimensions of fear of dental injections. J Am Dent Assoc 1997; 128: 756-766.

7. Kleinknecht R A, Thorndike R M, McGlynn F D, Harkavy J. Factor analysis of the dental fear survey with cross-validation. J Am Dent Assoc 1984; 108: 59-61.

8. Boyle C A Newton J T, Milgrom P. Who is referred for sedation for dentistry and why? Br Dent J 2009; 206: E12.

9. Allen E M, Girdler N M. Attitudes to conscious sedation in patients attending an emergency dental clinic. Prim Dent Care 2005: 12: 27-32.

10. Chanpong B, Haas D A, Locker D. Need and demand for sedation or general anesthesia in dentistry: a national survey of the Canadian population. Anesth Prog 2005; 52: 3-11.

11. Cutler C. Assessing patients' perception of self-care agency in psychiatric care. Issues Ment Health Nurs 2003; 24: 199-211.

12. Special Care Dentistry Association. SCDA definitions webpage. http://www.scdonline.org/displaycommon.cfm?an=1Etsubarticlenbr=148 (accessed 19 October 2009). 